\title{
Strain effect in silicon-on-insulator materials: Investigation with optical phonons
}

\author{
J. Camassel, L.A. Falkovsky* and N. Planes \\ Groupe d'Etudes des Semiconducteurs \\ UMR 5650 CNRS-UM2, \\ cc074Univer sité Montpellier 2 \\ Place E. Bataillon \\ F-34095 Montpellier cedex 5, France
}

(October 24, 2018)

\begin{abstract}
We report a detailed experimental and theoretical investigation of the effect of residual strain, and strain relaxation, which manifests itself at the $\mathrm{Si} / \mathrm{SiO}_{2}$ interfaces in commercial silicon-on-insulator (SOI) wafers. SOI material is made of a single-crystal silicon overlayer (SOL) on top of an insulator (buried $\mathrm{SiO}_{2}$ layer) sitting on a handle silicon wafer. Infrared reflectivity spectra show that the buried $\mathrm{SiO}_{2}$ layer relaxes continuously when thinning the SOL. At the same time the SOL surface roughness and the linewidth of optical phonons in Si near the $\mathrm{Si} / \mathrm{SiO}_{2}$ interface (probed by micro-Raman specroscopy) increase. In the as-delivered wafers, this comes from a slight expansion of $\mathrm{Si}$ on both sides of the buried $\mathrm{SiO}_{2}$ layer which, conversely, is compressed. Thinning the SOL modifies these initial equilibrium conditions. To get quantitative results, we have modeled all our Raman spectra using a theory of inhomogeneous shift and broadening for optical phonons, which takes into account the phonon interaction with the static strain fluctuations. From the variation of linewidth versus interface distance, we have found that the mean squared
\end{abstract}


strain continues to relax in the bulk of the wafer through a depth on the order of several $\mu \mathrm{m}$. We also show that the SOL surface roughness is related to strain fluctuations near the $\mathrm{Si} / \mathrm{SiO}_{2}$ interfaces.

63.20Mt, 78.30.-j, 79.60Jv

Typeset using REVTEX 


\section{INTRODUCTION}

Silicon-On-Insulator (SOI) technology emerged in the early 80s and became increasingly popular [1], [2]. Initially limited to space and military applications where radiation hardness is more important than cost and performance, SOI material was rapidly considered for PD (Partially Depleted) and FD (Fully Depleted) C-MOS (Complementary-Metal Oxide Semiconductor) technology. The net consequence is that IBM announced recently massproduction of ICs (Integrated Circuits) on SOI using PD-MOS architectures [3].

The main reason for success in the case of ICs on SOI is that unlike conventional bulk silicon they do not have any direct electrical connection between the active devices and the underlying substrate. This renders easier the production of low power / low voltage / high frequency ICs. However, since FD-MOS remains difficult to master, there is still room for deep scientific interest. In this work, we report a detailed consideration of the strain and strain relaxation effects which appear in such non-traditional bonded silicon substrates.

Whatever the manufacturing technology, all standard SOI wafers have the appearance shown in Fig. 1. On top is a thin film of single-crystalline silicon, next a buried oxide film and, finally, a thick (handle) silicon wafer. Usually the Silicon-OverLayer (SOL) is $0.2 \mu \mathrm{m}-$ thick and the Buried-OXide (BOX) is $0.4 \mu \mathrm{m}$-thick. Basically two different techniques can be used to produce the buried oxide layer. One is oxygen-ion implantation, which results in SIMOX (Separation by IMplantation of Oxygen). The second is hydrophilic bonding of two (previously oxidized) Si wafers, which results in BESOI (Bond and Etch back SOI) and Unibond [4]. Of course, the topmost SOL and the BOX should be defect-free, stress-free, and uniform in thickness. The handle Si wafer should be also defect-free.

The real situation departs from this ideal viewpoint. From a recent survey of literature data, the SOL surface roughness ranges from less than $2 \AA$ in Unibond to $5 \AA$ in high dose SIMOX, whereas the density of dislocations in the SOL runs from $100 \mathrm{~cm}^{-2}$ in Unibond to $10^{6} \mathrm{~cm}^{-2}$ in SIMOX. Since any direct bonding of two oxidized wafers (or any oxygen-ion implantation followed by a high temperature annealing step) must introduce a finite amount 
of stress at the interface [5], it is obvious that the primary source of defects in the SOL is the stress located at the $\mathrm{Si} / \mathrm{SiO}_{2}$ interface. Upon relaxation, this stress leads to the generation of dislocations which, in turn, affect the silicon parts of the material.

The study of the interaction between dislocations and a strain field is an old problem. In the recent years, it has been actively pursued (see, for instance, Refs. [6] - 8]), but many qualitative observations are still not satisfactorily understood. For instance, in recent work [9], we have reported some preliminary results concerning the strain in SOI, which need to be quantitatively discussed. We have used SIMOX and Unibond materials and, in both cases, started from $\sim 200 \mathrm{~nm}$ (nominal) SOL thickness. Then we thinned progressively down to $\sim 5 \mathrm{~nm}$ and observed three different phenomena: (i) a change in the $1050 \mathrm{~cm}^{-1}$ infrared reflectivity structure associated with the internal vibrational modes of the $\mathrm{SiO}_{4}$ tetrahedra located in the BOX, (ii) an increase in the SOL surface roughness probed by AFM (Atomic Force Microscopy) and, (iii) a variation in the linewidths of the optical phonons in the bulk of the handle Si-wafer as a function of the distance from the BOX/wafer interface. This was probed using micro-Raman spectroscopy. It was found that roughly speaking, given a specific SOL thickness, the linewidth becomes narrower as the distance from the interface increases. Conversely, given a distance, the linewidth was larger for the samples with the thinner overlayer. This behavior corresponds with surface roughness modification. The roughness of the free surface increases when the thickness of the overlayer decreases.

Since the same distance-to-interface dependence of the linewidth is found in different kinds of SOI (BESOI and SIMOX materials) it is necessary to propose a common model to explain the physical origin of this phenomenon. For instance, because the natural width of a Raman phonon line is mainly determined by the anharmonic decay of an optical phonon (with frequency $\omega_{0}$ ) into two phonons of opposite wave vectors, additional contributions near the interface could result from the decay into two surface phonons or, in the long-wave limit, into two acoustic surface Rayleigh waves. The excited surface phonons have frequencies $\omega_{0} / 2$ and wave vectors $k=\omega_{0} / 2 s_{t} \zeta$ (where $s_{t}$ is the transverse-acoustic velocity and the constant $\zeta$, of the order of 0.9 , depends on the elasticity constants of the material). Therefore, in 
the direction normal to the surface, the corresponding penetration depth $\delta \sim 1 / k$, being on the order of wavelength, is not larger than a few tens of lattice parameters $a$. Then the contribution to the Raman spectra must be of the order of $\delta / d_{s}$, where $d_{s}$ is the diameter of the laser spot. Such a small contribution is not observable in our experiments.

An alternative reason for broadening could be related to imperfections localized near the interface. In a single crystal only the phonon modes with a well defined wave-vector (close to the zone-center because the incident and scattered photons have very small momenta) can contribute to the Raman spectra. All departures from this "perfect crystal" conditions are known as a "relaxation of the momentum selection rule" [10. A simple (qualitative) description is usually obtained [11], by integrating the natural (Lorentzian) phonon line shape with a Gaussian distribution function of phonon states (the width of which is determined by the space scale of the imperfections). However, such a description does not explain the physical source of broadening.

In this work we challenge a model which takes quantitatively into account the scattering of phonons by structural imperfections. The central point is strain induced at the $\mathrm{Si} / \mathrm{SiO} 2$ interface because of the large difference in thermal expansion coefficients. This results in structural defects (or misfit dislocations) in the adjacent layers. Away from the interface the strain relaxes at some finite distance, involving new threading dislocations. The effect of the smooth part of the strain on the phonon frequencies can be compared with the influence of pressure. With this respect, our theory is consistent with the well known results for the pressure dependence of phonon frequencies. This was previously observed under homogeneous stress in Si, Ge and GaAS [12] or thin films of cubic SiC on Si [13], [14] and results in well-known phonon shift and splitting.

The specific effect of strain fluctuations is to give rise to a phonon scattering process which induces additional phonon width and shift. Despite the fact that the effect of disorder on vibrational modes has been studied for a long time [15], a detailed model has only been proposed recently. Taking into account the optical phonon scattering due to disorder, the theory describes the inhomogeneous phonon width associated with phonon scattering by the 
strain fluctuations and explains satisfactorily all relevant experimental results [14].

One important point to notice is that the most distinctive features of the optical-phonon scattering by static imperfections is the influence of the DOS singularity at the zone-center. If the optical phonon branch has a maximum (minimum) value $\omega_{0}$ at the zone-center, the cross section of the phonon-impurity scattering vanishes at $\omega=\omega_{0}$ and increases for $\omega^{2}<\omega_{0}^{2}$ $\left(\omega^{2}>\omega_{0}^{2}\right.$, respectively). Then the Raman line shape as a function of the frequency $\omega$ shows asymmetry, being broader on the low (high) frequency side. The asymmetry is sensitive to the dimensions of the disorder [16]. This important role of the "density-of-state" effect was noticed only recently for films subjected to laser heating [17] and explain satisfactory the final line shape observed in this work.

Our paper is organized as follows. To analyze the experimental data in detail, we discuss the theory of probing the strain by Raman spectroscopy in Sec. II. In Sec. II-A, we recapitulate the equation of motion of optical phonons in the long-wave approximation and show that the effect of homogeneous strain gives rise to the splitting and shift of the optical phonon triplet. In Sec. II-B, we show how the static strain fluctuations involve the phonon scattering and result in increased phonon linewidths. Since the increase in linewidth can be larger than the natural phonon width in the anharmonic decay channels, we consider the effect of fluctuations self-consistently using Dyson's equation for the phonon Green's function. We obtain the inhomogeneous broadening and shift in terms of the strain correlation function. Supposing that the main strain effect is connected to the dislocations, a two-dimensional form of the correlation function is used. In Sec. II-C, we discuss the effect of surface roughness. In Sec. III, we summarize all experimental details and compare them with theory. In Subsec. III-A we discuss the results obtained from the infrared spectra collected as a function of SOL thickness for various SOI wafers. We show that they give evidence of residual permanent strain in the as-delivered wafers. Within the BOX, the strain relaxes more and more when the SOL thickness decreases. This is independent of the SOI wafer fabrication process as well as the thinning method. In Subsec. III-B, we show that this decrease of residual strain in the BOX correlates directly with an increase in the Raman 
linewidth in the handle silicon wafer. This is observed from micro-Raman spectra collected on the cleaved edges of wafers. Near the interface Raman spectra show that the threefold degeneracy of the $k \simeq 0$ optical phonon is split indicating a large (local) strain. We deduce a noticeable inhomogeneous contribution to the linewidth, which extends down to several micrometers. Finally, we show that the surface SOL roughness is directly related to the strain fluctuations near the BOX/wafer interface.

\section{THEORY}

\section{A. Summary of strain effect on optical phonons}

In $\mathrm{Si}$, at the $\Gamma$ point of the Brillouin zone the optical phonon displacements $u_{i}(\mathbf{r}, \omega)$ (where $i=x, y, z)$ belong to the threefold representation $\Gamma_{25}^{\prime}$ of the diamond group $O_{h}^{7}$. The interaction of these phonons with the static strain $\varepsilon_{i j}(\mathbf{r})$ corresponds to the third order terms (which are linear in $\varepsilon_{i j}(\mathbf{r})$ and bilinear in $\left.u_{i}(\mathbf{r}, \omega)\right)$ in the total energy expression. The first derivatives of the total energy with respect to $u_{i}(\mathbf{r}, \omega)$ give then the equation of motion

$$
\left(\left(\omega_{0}^{2}+s^{2} \Delta-i \omega \Gamma^{(i n t)}-\omega^{2}\right) \delta_{i j}+V_{i j}(\mathbf{r})\right) u_{j}(\mathbf{r}, \omega)=0
$$

where the zone-center optical phonon frequency $\omega_{0}=520 \mathrm{~cm}^{-1}$ for Si and the corresponding natural width $\Gamma^{(i n t)}$ is about $3 \mathrm{~cm}^{-1}$. The optical branches in cubic crystals are dispersive and, for instance, the dispersion of the $x$-polarized optical branch has the form: $\omega^{2}=$ $\omega_{0}^{2}-s_{1}^{2} k_{x}^{2}-s_{2}^{2}\left(k_{y}^{2}+k_{z}^{2}\right)$. The neutron scattering experiments give an estimate of the dispersion parameters $s_{1} \approx s_{2} \approx 0.8 \cdot 10^{5} \mathrm{~cm} / \mathrm{s}$ for $\mathrm{Si}$. This shows that the dispersion term can be written in a more simple (isotropic) form $s^{2} \partial^{2} / \partial x_{i}^{2}$ in the vicinity of the branch maximum at the Brillouin-zone center.

The interaction $V_{i j}(\mathbf{r})$ describes the changes in "spring constants" due to strain $\varepsilon_{i j}(\mathbf{r})$ :

$$
V_{x x}(\mathbf{r})=\lambda_{x x x x} \varepsilon_{x x}(\mathbf{r})+\lambda_{x x y y}\left[\varepsilon_{y y}(\mathbf{r})+\varepsilon_{z z}(\mathbf{r})\right] \quad \text { and } \quad V_{x y}(\mathbf{r})=\lambda_{x y x y} \varepsilon_{x y}(\mathbf{r})
$$

where we take into account the fact that only three independent components $\lambda_{i j k l}$ exist in a cubic crystal. The other elements of $V_{i j}(\mathbf{r})$ can be obtained by circular permutations of 
the indices in Eq. (2). It is evident that they are of order $\omega_{0}^{2}$. From experiments with homogeneous stress [12] we find the coefficient values $\lambda_{x x x x} \equiv p=-1.6 \omega_{0}^{2}, \lambda_{x x y y} \equiv q=$ $-2.2 \omega_{0}^{2}$ and $\lambda_{x y x y} \equiv 2 r=-1.3 \omega_{0}^{2}$, where the generally accepted notation $p, q, r$ is used.

Because of the strain fluctuations, all quantities have to be averaged over physically small distances (which are still large on a microscopic scale). The averaged strain $\left\langle\varepsilon_{i j}\right\rangle$ conserves cubic symmetry in the plane parallel to the interface, but depends smoothly on the distance to the interface. In Fig. 1 this is chosen as the $z$-direction. We do not write this dependence in explicit form. The average components are such that: $\left\langle\varepsilon_{x y}\right\rangle=\left\langle\varepsilon_{x z}\right\rangle=\left\langle\varepsilon_{y z}\right\rangle=0$ and $\left\langle\varepsilon_{x x}\right\rangle=\left\langle\varepsilon_{y y}\right\rangle$, which gives for the shifted phonon frequencies at the Brillouin-zone center

$$
\omega_{z}^{2}(k=0)=\omega_{0}^{2}+\lambda_{x x x x}\left\langle\varepsilon_{z z}\right\rangle+2 \lambda_{x x y y}\left\langle\varepsilon_{x x}\right\rangle
$$

for the phonon polarization normal to the interface and

$$
\omega_{x}^{2}(k=0)=\omega_{y}^{2}(k=0)=\omega_{0}^{2}+\left(\lambda_{x x x x}+\lambda_{x x y y}\right)\left\langle\varepsilon_{x x}\right\rangle+\lambda_{x x y y}\left\langle\varepsilon_{z z}\right\rangle
$$

for the phonons polarized in the plane of the interface. This is nothing but the well-known phonon spliting into a $z$-singlet and an $x y$-doublet under the effect of a homogeneous strain [12] - 14].

\section{B. Effect of strain fluctuations on the optical-phonon linewidth}

The contribution to the linewidth arises in the second-order perturbation theory for the strain components. It could be calculated using "the golden rule" of quantum mechanics. However, due to the singularity in the phonon density-of-states at $\omega=\omega_{0}$, this contribution is strongly frequency-dependent and may be larger than the natural phonon linewidth. Therefore we calculate it self-consistently using a Green's function technique [18. For the first-order Raman scattering, the corresponding cross section is bilinear in the phonon displacements. Then the Raman cross section

$$
\frac{d \sigma}{d \omega^{(s)} d \Omega^{(s)}} \propto \frac{e_{\beta}^{(i)} e_{\gamma}^{(s)} e_{\beta^{\prime}}^{(i)} e_{\gamma^{\prime}}^{(s)} g_{\alpha \beta \gamma} g_{\alpha^{\prime} \beta^{\prime} \gamma^{\prime}}}{1-\exp \left(-\hbar \omega / k_{B} T\right)} \operatorname{Im} D_{\alpha \alpha^{\prime}}(\mathbf{k}, \omega)
$$


is determined by the imaginary part of the phonon Green's function $D_{\alpha \alpha^{\prime}}(\mathbf{k}, \omega)$, where $\omega$ and $\mathbf{k}$ have the sense of frequency and momentum transfers, $e_{\beta}^{(i)}$ and $e_{\gamma}^{(s)}$ are the polarization vectors of the incident and scattered photons, respectively, and $g_{\alpha \beta \gamma}$ is the $\beta \gamma$-component of the Raman polarizability tensor for the $\alpha$-phonon branch. It is easy to see that, for the $\Gamma_{25}^{\prime}$ representation, the tensor $g_{\alpha \beta \gamma}$ is completely antisymmetric and reduces to the component $g_{x y z}$. This means that, when the incident and scattered fields are polarized along the $x$ and $y$ axes, respectively, only $z$-polarized phonons can be excited.

The phonon Green's function has to be averaged over the strain distribution. The calculation has been performed in details in a previous work [14] and we shall simply summarize the results. The average phonon Green's function has the diagonal form:

$$
D_{j j}(\mathbf{k}, \omega)=\left(\Omega_{j}^{2}(\mathbf{k}, \omega)-s^{2} k^{2}-i \omega \Gamma_{j}(\mathbf{k}, \omega)-\omega^{2}\right)^{-1}
$$

where the unknown real functions $\Omega_{j}(\mathbf{k}, \omega)$ and $\Gamma_{j}(\mathbf{k}, \omega)$ obey the system of Dyson's equations

$$
\Omega_{j}^{2}(\mathbf{k}, \omega)-\omega_{0}^{2}-\left\langle V_{j j}\right\rangle-i \omega\left(\Gamma_{j}(\mathbf{k}, \omega)-\Gamma^{(i n t)}\right)=-\sum_{q} \frac{W_{j m}(\mathbf{q}-\mathbf{k})}{\Omega_{m}^{2}(\mathbf{q}, \omega)-s^{2} q^{2}-i \omega \Gamma_{m}(\mathbf{q}, \omega)-\omega^{2}} .
$$

The average interaction $\left\langle V_{j j}\right\rangle$ is defined in Eqs. (2) - (4) and the correlation function in real space is

$$
W_{j m}\left(\mathbf{r}-\mathbf{r}^{\prime}\right)=\left\langle\delta V_{j m}(\mathbf{r}) \delta V_{m j}\left(\mathbf{r}^{\prime}\right)\right\rangle
$$

with $\delta V_{j m}(\mathbf{r})=V_{j m}(\mathbf{r})-\left\langle V_{j m}\right\rangle$.

Several points should be emphasized. First, one can see from Eqs. (5) and (6) that the line center as a function of the frequency transfer $\omega$ is determined by the equation $\Omega_{j}^{2}(\mathbf{k}, \omega)-s^{2} k^{2}=\omega^{2}$, where $\mathbf{k}$ is the momentum transfer. Here we can put $k=0$, since the photon wave vector is small. Second, in the limiting case $\Gamma_{j}(\mathbf{k}, \omega) \rightarrow 0$, the bypass around the pole in the right-hand side of Eq. (7) gives the Dirac delta-function $\delta\left(\Omega_{m}^{2}(\mathbf{q}, \omega)-s^{2} q^{2}-\omega^{2}\right)$. We then arrive at the well-known golden rule and see that the strain fluctuations make a 
contribution into the linewidth only for $\omega^{2}<\Omega_{m}^{2}$, i. e. on the low-frequency side of the Raman line. The opposite situation occurs when the phonon branch has a minimum at the center of the Brillouin zone [16] but, in both cases, the line shape shows asymmetry.

\section{Simplest model of two-dimensional disorder}

Equations (7) can be simplified if we assume that the correlator $W_{j m}(\mathbf{q})$ has a constant value $W_{j m}(\mathbf{q}=0)$ in the region $q<1 / r_{0}$ and vanishes elsewhere. On the other hand, the strain fluctuations may be considered as imperfections where $W_{j m}(\mathbf{q}=0)$ is proportional to the concentration of imperfections and $r_{0}$ is the domain size in which a phonon interacts with the imperfection. The final form of Eqs. (7) depends on the dimensionality of the imperfections. In our previous work [14] we have concluded that the imperfections involved by interfaces are mainly two-dimensional, i. e. that they behave like dislocations. In that case, $r_{0}$ is the dislocation core and the correlation function $W(\mathbf{r})$ in Eq. (8) depends on the two-dimensional vector $\mathbf{r}_{\perp}$. Then Eqs. (7) have the form:

$$
\begin{array}{r}
\Omega_{j}^{2}(\omega)-\omega_{0}^{2}-\left\langle V_{j j}\right\rangle-i \omega\left(\Gamma_{j}(\omega)-\Gamma^{(i n t)}\right) \\
=\omega_{0} B_{j m}\left(\frac{1}{2} \log \frac{\left(s^{2} / r_{0}^{2}+\omega^{2}-\Omega_{m}^{2}(\omega)\right)^{2}+\omega^{2} \Gamma_{m}^{2}(\omega)}{\left(\omega^{2}-\Omega_{m}^{2}(\omega)\right)^{2}+\omega^{2} \Gamma_{m}^{2}(\omega)}\right. \\
\left.-i \arctan \frac{\omega^{2}-\Omega_{m}^{2}(\omega)+s^{2} / r_{0}^{2}}{\omega \Gamma_{m}(\omega)}+i \arctan \frac{\omega^{2}-\Omega_{m}^{2}(\omega)}{\omega \Gamma_{m}(\omega)}\right),
\end{array}
$$

where $B_{j m}=W_{j m}\left(\mathbf{k}_{\perp}=0\right) / 4 \pi s^{2} \omega_{0}$, and the sum can be taken over two split modes, which are the normally polarized singlet and the doublet parallel to the interface. Notice that the functions $\Omega_{j}(\omega)$ and $\Gamma_{j}(\omega)$ are independent of $\mathbf{k}$ (we omit $\mathbf{k}$ in the arguments) because of

its smallness in comparison to the value $\sqrt{\omega_{0} \Gamma} / s \simeq(\pi / a) \sqrt{\Gamma / \omega_{0}}$ which is essential in the integral (7). Again $a$ is the lattice parameter.

Let us estimate $B_{j m}$. According to Eqs. (11) and (2), $V(r=0)=\lambda \varepsilon \sim \omega_{0}^{2} \varepsilon$, where we omit the tensor indices. We have for the Fourier component (8) $W_{j m}(k=0) \sim 2 \pi \omega_{0}^{4}(\delta \varepsilon)^{2} r_{0}^{2}$ and for the phonon-strain scattering probability

$$
B \sim \omega_{0}^{3}\left(r_{0} \delta \varepsilon / s\right)^{2}
$$


where $\delta \varepsilon$ is the strain fluctuation.

If we assume that the strain fluctuations are mainly induced by dislocations, then

$$
B=c v^{2}(k=0) / 4 \pi \omega_{0} s^{2}
$$

In this expression, $c$ is the dislocation concentrations, $v(k=0)=g r_{0}^{2} \omega_{0}^{2}$ is the phonondislocations interaction where $g$ is a dimensionless constant of the order of unity and $r_{0}$ is the radius of a dislocation core.

In the following we will solve numerically Eqs.(9) in $\Omega_{j}(\omega)$ and $\Gamma_{j}(\omega)$ to find the values of $B_{j m}$ and $r_{0}$ which give a fit of Eqs. (5) and (6) to the experimental Raman spectra. Notice that the effect of the strain fluctuations on the line shape is qualitatively evident from Eqs.(9). For instance, if there is only one phonon mode, its width $\Gamma(\omega)$ is governed by the equation

$$
\Gamma(\omega)-\Gamma^{(i n t)}=\frac{\omega_{0} B}{\omega}\left(\arctan \frac{\omega^{2}-\Omega^{2}(\omega)+s^{2} / r_{0}^{2}}{\omega \Gamma(\omega)}-\arctan \frac{\omega^{2}-\Omega^{2}(\omega)}{\omega \Gamma(\omega)}\right) .
$$

Let us consider the case of small $r_{0}\left(r_{0}<s / \sqrt{\omega \Gamma}\right)$. The terms in the right-hand side of Eq. (12) compensate for each other on the high-frequency side $\left(\omega^{2}>\Omega^{2}\right)$ of the Raman line, where the line has a nearly Lorentzian form with a width $\Gamma \simeq \Gamma^{(\text {int })}$. For the lowfrequency side $\left(\omega^{2}<\Omega^{2}\right)$, these two terms give a contribution to the linewidth which is proportional to the squared strain fluctuations. For the situation of a minimum in the phonon branch at the center of the Brillouin zone, the Raman line drops more rapidly on the low-frequency side. This effect has origin in the singularity of the phonon densityof-states at the branch extremum. In the opposite case, $r_{0} \gg s / \sqrt{\omega \Gamma}$, the lineshape is symmetric but non-Lorentzian.

Since $\Gamma(\omega)$ is $\omega$-dependent, we must connect its value with the full width at half maximum (FWHM) of the experimental spectra. The simplest way is to use $\Gamma(\omega)$ at the line peak, where the equation $\Omega(\omega)=\omega$ is satisfied. In the case where $\Gamma$ is independent of $\omega$, this definition gives the FWHM. The first term in the parenthesis Eq. (12) remains only at the line peak. Finally, because the difference between the line positions $\Omega(\omega)$ and $\omega_{0}$ (including and ignoring the effect of disorder, respectively) is small, we obtain for $\Gamma$ at the line peak 


$$
\Gamma-\Gamma^{(i n t)}=B \arctan \frac{s^{2}}{r_{0}^{2} \omega_{0} \Gamma}
$$

Notice that, in the limiting case $r_{0} \ll s / \sqrt{\omega \Gamma}$, Eq.(13) coincides with the result of the perturbation theory $\Gamma=\Gamma^{(i n t)}+\pi B / 2$. In the opposite case $\arctan \left(s^{2} / r_{0}^{2} \omega_{0} \Gamma\right)=s^{2} / r_{0}^{2} \omega_{0} \Gamma$ and we arrive at a quadratic equation which gives

$$
\Gamma=\frac{\Gamma^{(i n t)}}{2}+\sqrt{\left(\frac{\Gamma^{(i n t)}}{2}\right)^{2}+\frac{B s^{2}}{\omega_{0} r_{0}^{2}}}
$$

This shows that, in the general case, the effect of disorder which is represented by the parameter $\mathrm{B}$, is not simply additive with the natural width $\Gamma^{(i n t)}$.

\section{Surface roughness induced by dislocations}

Because of the interface strain and related dislocations, there must be a finite surface roughness contribution. Due to the very complicated nature of the interactions between the surface and dislocations it seems impossible to reach a high degree of sophistications in this problem. However, we can estimate the mean roughness using a qualitative approach. We suppose that the strain has large fluctuating components and calculate the mean square roughness.

Any rough plane may be conceived as a set $\zeta(\mathbf{s})=\sum_{n} b\left(\mathbf{s}-\mathbf{s}_{n}\right)$ of randomly located "hills" at points $\mathbf{s}_{n}$, where $\mathbf{s}$ is a two-dimensional vector of the free surface plane. If we

suppose that the hills originate from dislocations, the height $b$ must be on the order of the Burgers vector $b_{0}$. We specify $l$ as the size of the hills (i.e. the domain size where the function $b\left(\mathbf{s}-\mathbf{s}_{n}\right)$ has a nonzero value) and, by averaging over the distribution of points $\mathbf{s}_{n}$, we obtain the roughness correlation function

$$
\zeta_{2}\left(\mathbf{s}-\mathbf{s}^{\prime}\right)=<\zeta(\mathbf{s}) \zeta\left(\mathbf{s}^{\prime}\right)>=c \sum_{q}|b(\mathbf{q})|^{2} e^{i \mathbf{q}\left(\mathbf{s}-\mathbf{s}^{\prime}\right)}
$$

where $b(\mathbf{q}) \sim b_{0} l^{2}$ is the Fourier component of the function $b(\mathbf{s})$ and $c$ is the hill concentration at the surface. 
The mean square roughness is given by $\zeta_{2}\left(\mathbf{s}=\mathbf{s}^{\prime}\right) \simeq c b_{0}^{2} l^{2}$ which is connected to the strain relaxation by the dislocation concentration $c$. Of course some initial roughness $\zeta_{0}^{2}$ does exist, which is not strain-dependant. Taking both contributions into account, we write for the effect of strain on the surface roughness

$$
\zeta^{2}=\zeta_{0}^{2}+c b_{0}^{2} l^{2}
$$

This expression and Eqs. (11), (13) enable us to compare the change in roughness with the phonon linewidth. We get

$$
\Gamma-\Gamma^{(i n t)}=A\left(\zeta^{2}-\zeta_{0}^{2}\right) \arctan \frac{s^{2}}{r_{0}^{2} \omega_{0} \Gamma},
$$

where $A=g^{2} r_{0}^{4} \omega_{0}^{3} / 4 \pi s^{2} b_{0}^{2} l^{2}$.

\section{EXPERIMENTAL RESULTS AND COMPARISON WITH THEORY}

Most experimental details have already been given in [9] and will not be repeated here. To summarize briefly, three different (commercial) 4-inch wafers from SOITEC [⿴囗⿰丿㇄口] were considered. One wafer was high dose SIMOX and two were Unibond. In all three cases, the nominal SOL thickness was $0.2 \mu \mathrm{m}$. In two cases (one Unibond wafer and the SIMOX wafer), a wet SO (Sacrificial Oxidation) process was used. This resulted in two series of samples denoted A and B, respectively. In order to evaluate the effect of the repetitive high temperature oxidation steps (at $1050 \mathrm{C}$ ) on the final results, a low temperature oxygenassisted IBE (Ion Beam Etching) process was applied to the second Unibond wafer. This resulted in the third series of samples (samples C).

\section{A. Influence of the SOL thickness on the IR reflectivity spectra}

To control the SOL thickness [19] infrared reflectivity spectra were systematically collected at room temperature with a Brucker IFS 66v Fourier Transform Spectrometer fitted with a DTGS detector. Measurements were made in the spectral range 400 to $7500 \mathrm{~cm}^{-1}$ 
and a standard "near normal incidence" configuration (finite value of the angle of incidence close to $20^{\circ}$ ) was used. The direction of incidence was in the $y z$-plane of Fig. 1 and no attempt was made to polarize the incident light.

Apart from the change in interference patterns which gives the final sample thicknesses, a strong effect was found in the $1000-1100 \mathrm{~cm}^{-1}$ range. This is the narrowing of the $\mathrm{SiO}_{2^{-}}$ related feature displayed in Fig. 2 for samples A. It has nothing to do with the change in the interference pattern, but correlates with the change in SOL thickness. Notice that there is a large effect when reducing the SOL from 203 to 95 and $42 \mathrm{~nm}$. Notice also that, starting from $42 \mathrm{~nm}$, it seems to saturate.

The $1070-1080 \mathrm{~cm}^{-1}$ doublet structure is well known. It manifests itself in every silicate-like material (not only in crystals, [20]- 223], but also in vitreous silica [24] or, even, in sol-gel derived glasses [25]) and corresponds with internal vibrations of the building $\mathrm{SiO}_{4}$ tetrahedra. It is very important to notice the stability of the corresponding frequency which does not change in going from $\beta$ - to $\alpha$-quartz [22] or, even, when $\mathrm{Si}$ is replaced by $\mathrm{Al}$ or $\mathrm{P}$ in $\mathrm{AlPO}_{4}$, which is the ternary analog of quartz [23]. In the literature it has been classified as an AS (Asymmetric Stretching) internal mode [24], 25] in which one oxygen atom bonded to two neighboring Si moves parallel to the Si-Si direction. This oxygen motion is strongly infrared active and, despite the lack of long-range ordering, in many cases the associated LO frequency resolves around $1260 \mathrm{~cm}^{-1}$. Due to the finite value of the angle of incidence, this feature appears also in the present work.

Concerning Fig. 2, a second point should be emphasized. In the specific case of a thin $\mathrm{SiO}_{2}$ film on $\mathrm{Si}$, one expects two equivalent in-plane $x$ - and $y$-modes and only one out-of-plane $z$-mode. In other words, because we are dealing with a thin layer in which the threefold degeneracy is lifted, one expects to find a doublet structure with a 1 to 2 intensity ratio. This is exactly what is found. Starting from one broad (unresolved) feature for nominal SOL thickness $200 \mathrm{~nm}$, one goes rapidly to two distinct vibrational modes when the SOL thickness reduces below $40 \mathrm{~nm}$. From the corresponding intensity ratio, we deduce that the $z$-mode appears lower. Since the average energy difference with respect to the 
$x, y$-modes comes from the thermal strain, the strain is compressive. Making the length of the $\mathrm{Si}$-O bonds oriented in the $x y$-plane shorter, it shifts the $x, y$-modes to higher energy. Of course there are some finite deviations with respect to the average energies and, since we find evidence of a clear re-ordering (within the thin dielectric film) when thinning the SOL, we must conclude that the SOI system is not at all rigid but, instead, at the atomic scale constitutes a rather flexible medium.

Since the simplest way to shift the frequency of an internal mode is to change the bond length, our results suggest that we are dealing with oxide relaxation. To explain from the very beginning the narrowing of the Si-O related feature, one must assume an initial (thermal strain induced) distortion which relaxes when the SOL thickness decreases. Starting from a broad feature $\left(\sim 100 \mathrm{~cm}^{-1}\right)$ for the as-delivered material (broad distortion of the equilibrium bond length) the narrowing of the Si-O bond signature demonstrates a reordering of the $\mathrm{Si}-\mathrm{O}$ distances as the SOL thickness decreases. This is true from the very beginning, when thinning the SOL from 200 to $100 \mathrm{~nm}$, and does not depend on the type of material investigated. This observation has been checked on SIMOX (series B) and similar results were found.

Finally, to check that this was not due to any parasitic effects of the high temperature SO steps, we repeated the thinning process using oxygen-assisted IBE (series C). Again, similar results were found. This lends support to the proposition that we are dealing with real and intrinsic properties: the experimental improvement found in the oxide relies on an internal stress relaxation mechanism, where the stress originates only from the difference in thermal expansion coefficients between $\mathrm{Si}$ and $\mathrm{SiO}_{2}$ ( $\sim 80 \%$ at room temperature). There is no artifact associated with the high temperature SO steps and, to the best of our knowledge, this provides the first clear experimental evidence that all SOI materials are plastic systems, soft enough to relax when the SOL thickness decreases.

To estimate the relaxation we write the interatomic potential energy proportionally to $r^{-n}$, where $n$ should be of the order of 5 for a typical oxide. Because the relative change of the vibration frequency is $\delta \omega / \omega=(n+2) \delta r / 2 r$ and the linewidth of the reflectivity signal 
changes by around $50 \mathrm{~cm}^{-1}$ when thinning the SOL, we deduce that the average change in $\mathrm{Si}-\mathrm{O}$ bond length is a reduction of about $1 \%$.

\section{B. Raman investigation of nominal and thinned SOI}

We have mainly shown that, because of internal stress relaxation, the BOX properties improve when thinning the SOL. Now we consider what happens in the silicon overlayer and wafer. First we investigated in details the Si handle-wafer by using micro-Raman spectroscopy. We use a Jobin-Yvon ISA T 64000 spectrometer fitted with an Olympus microscope and a cooled CCD detector. The $488 \mathrm{~nm}$ line of a Spectra-Physics argon-ion laser was used as the excitation frequency. To achieve in-depth resolution, a transverse backscattering configuration was used [14] with both the incident and scattered light propagating along the $x$-direction of Fig.1. Because of the large experimental aperture we probe, both, $y$ and $z$ polarized phonons.

Typical results are shown in Figs. 3 to 6 for the series of samples A (Unibond). Fig. 3 corresponds to nominal material with SOL thickness 203 nm (standard value), Fig. 4 with 42 nm (thinned), Fig. 5 with 11 nm (thinned) and, finally, Fig. 6 with 5 nm. Given a specific SOL thickness, the different spectra correspond with the different laser spot distance from the wafer surface. To quantify in full details the different contributions, they have been fitted using the theory of phonon interaction with static strain fluctuations presented in

Sec. II. The results are shown as solid lines on the different figures. The complete series of parameters is given in Table 1.

The fits with Eq. (5) and Eq. (6) were made simultaneously for all spectra. The best value of the parameter $r_{0} \omega_{0} / s=16$ gives a reasonable value for the correlation radius (or the dislocation core) $r_{0}=1.3 \AA$. Eqs. (9) were solved numerically to obtain the matrix elements $B_{i j}$, which determine the inhomogeneous linewidth and the shift of the lines. One example of the solution (functions $\Gamma_{j}(\omega)$ and $\Omega_{j}(\omega)-\omega_{0}-\left\langle V_{j j}\right\rangle / 2$ ) is presented in the right panel of Fig. 4 for the $z$-phonon $(j=z)$. The resulting values (i.e. the matrix elements $B_{x x}$ and 
$B_{z z}$ ) are given in Table I for samples A.

Every time, focusing far from the interface (a typically value is around $5 \mu \mathrm{m}$ for the complete series of samples) one finds an identical (standard) "bulk" Raman linewidth for the silicon handle wafers. In the following this is taken as "reference material" and the line position for the reference material $\left(520 \mathrm{~cm}^{-1}\right)$ is shown by the vertical dash-dotted line as a guide for the eye. Moving toward the surface (first to $2 \mu \mathrm{m}$ and, then, to $1 \mu \mathrm{m}$ ) has two consequences: i) one shifts significantly the phonon frequency to lower energy and ii) one broadens considerably the width of the Raman peak, especially in the cases of the thinner SOL. This is evident starting from $42 \mathrm{~nm}$.

A first contribution to the phonon lineshift towards the low frequencies comes as a result of the expansion of the $\mathrm{Si}$ lattice near the $\mathrm{Si} / \mathrm{SiO}_{2}$ interface [12], [14]. This homogeneous shift is described by Eqs. (3) - (四) but, because of the small energy difference, the strain-induced splitting into a singlet and a doublet states resolves only for the thinnest SOL thicknesses (5 and $11 \mathrm{~nm}$, respectively: see Table 1 ).

The second contribution comes from the strain relaxation mechanism which manifests itself experimentally when probing at different positions along the $z$-direction. It is frequency dependent and gives the inhomogeneous linewidth and shift contributions displayed in the right panel of Fig. 4. Notice that, when focussing on the active part of the SOI wafers (upper part of the handle Si wafer) the inhomogeneous linewidth and shift contributions at the line center become very large. They range from $1.1 \mathrm{~cm}^{-1}$ (width) and $+0.14 \mathrm{~cm}^{-1}$ (shift) for the $42 \mathrm{~nm}$ SOL sample to $6.1 \mathrm{~cm}^{-1}$ and $+0.36 \mathrm{~cm}^{-1}$, respectively, for the $5 \mathrm{~nm}$ SOL sample (see again Table I). The inhomogeneous contribution to the line-shift remains however smaller than the homogeneous ones: $-0.9 \mathrm{~cm}^{-1}$ for the $42 \mathrm{~nm}$ SOL sample and -1.2 $\mathrm{cm}^{-1}$ for the $5 \mathrm{~nm}$ SOL sample.

Similar results are found for SIMOX. They are displayed in Fig. 7 for the SOL thickness $50 \mathrm{~nm}$. The natural width is slightly larger (3.4 instead of $3.1 \mathrm{~cm}^{-1}$ for Unibox) but the correlation radius has the same value $r_{0} \omega_{0} / s=16$. The main distinction is that the depth of strain relaxation is larger: there is still a noticeable difference in the linewidths at distances 
5 and $20 \mathrm{~nm}$ to the interface (see Table II). Finally, some homogeneity at the interface is found. Two spectra (last rows of Table II) collected from different points at the interface show practically the same characteristics. However other regions were detected, where more dramatic differences do exist. We shall come back to this point in Sec. IV (Conclusions).

To summarize this section, we have found from our analysis of the linewidth variation versus distance that all strain-induced effects (like the homogeneous and inhomogeneous shifts and broadening) have a maximum near the $\mathrm{Si} / \mathrm{SiO}_{2}$ interfaces. Because they depend directly on the SOL thickness, they should affect primarily the thinned SOLs. The surprising effect is that they affect also the upper part of the handle wafers and relax through a depth on the order of several $\mu \mathrm{m}$.

\section{Surface roughness and Raman width}

From the Raman investigations, we have found that the morphology of the upper part of every (nominal and thinned) SOI wafer experiences a complex stress distribution. Moreover, the strain in the wafer near the BOX/wafer interface becomes larger when thinning the SOL. In this section we show that independent evidence can be found from the consideration of root mean square roughness of the SOL surface. We reported already that there is a strong degradation of the surface when the SOL thickness decreases [9]. Typically, decreasing the SOL thickness from 203 to $5 \mathrm{~nm}$, one increases the mean square AFM (Atomic Force Microscopy) roughness from 1.5 to $4 \AA$ RMS.

To show that the Raman width and mean surface roughness have the same origin (inhomogeneous strain and dislocations) we show in Fig. 8 a correlation of the Raman linewidths $\left(\mathrm{cm}^{-1}\right)$ obtained at the surface of wafers A with different SOL thickness with AFM data $(\AA)$. The solid lines are plots of Eq. (15). The experimental values are shown by dots. A single parameter $A=2.1 \mathrm{~cm}^{-1} \AA^{-2}$ was chosen to fit the values of the $z$-phonon width and roughness in the case of the SOL thickness of $5 \mathrm{~nm}$. Notice that the doublet splitting resolves only for the 5 and $11 \mathrm{~nm}$ SOL thickness. The value $A=0.28 \mathrm{~cm}^{-1} \AA^{-2}$ for the $x$-phonon 
was obtained in the comparison of the interaction constants $B_{x x}$ and $B_{z z}$ from Table I. From these data, it is interesting to estimate the parameter $l$ of the surface roughness using this value of $\mathrm{A}$ ( $r_{0}$ is obtained in this work and $s$ is known). Taking into account the expression of $A$ given after (15) and putting $g=1$ and $b_{0}=1 \AA$, we get a very reasonable value $l=88 \AA$ for the average diameter of the hills. The main difference from one thinning process to the next one is in the initial roughness. With this respect, all results obtained using SO $(1.2 \AA$, series A) appear better than the one obtained with IBE (2 A, series C).

\section{CONCLUSIONS}

It has been known for a very long time that any direct bonding of two oxidized wafers (or any oxygen-ion implantation followed by a high temperature annealing step, for instance) introduces a finite stress at the $\mathrm{Si} / \mathrm{SiO}_{2}$ interface. This comes directly from the difference in thermal expansion coefficients between $\mathrm{Si}$ and $\mathrm{SiO}_{2}\left(2.6 \cdot 10^{-6} \mathrm{~K}^{-1}\right.$ and $0.56 \cdot 10^{-6} \mathrm{~K}^{-1}$, respectively [2]). Because this constitutes a hard point of the technology, low temperature bonding using low viscosity oxides like BPSG (BoroPhosphoroSilicate Glass) or SOG (Spin on Glass) are under active development. In some cases they will offer alternative solutions. The problem of process-induced defects is totally different. To the best of our knowledge, it is much less documented. For instance there is no clear report of a critical SOL thickness, similar to the critical layer thickness encountered in hetero-epitaxy. Above such a critical thickness, the SOL material would be homogeneously strained but stable. It would strongly relax around that thickness, with a maximum in inhomogeneous behavior. Finally, it would be heavily strained, but more homogeneous and again stable below that value. Our experimental results suggest that such a critical thickness does exist. It is about 40 to $50 \mathrm{~nm}$ and corresponds with the experimental situations illustrated in Fig. 2 (BOX), Fig. 9 (Unibond) or Fig. 10 (SIMOX). In all three cases we find a critical regime where, simultaneously, the BOX achieves a better relaxation while there is a very large inhomogeneity in the SOL.

The spectra displayed in Figs. 9 and 10 (solid lines are again theoretical curves) could be 
understood in the terms of phonon splitting of the order of 7 to $15 \mathrm{~cm}^{-1}$ due to a very high local strain in some finite parts (or domains) at the interface.

To summarize, we have evidenced in this work that SOI is not a perfectly strain-relaxed system. It behaves more like a balanced-strain structure schematically drawn in Fig. 11. The interesting point is that: i) compressive strain (about $1 \%$ in absolute value) is always present in the buried oxide of as-delivered SOI wafers. This strain diminishes when the SOL thickness decreases; ii) on contrary, tensile strain exists in the silicon overlayer. Dislocations near the interface release the strains and manifest themselves in final surface roughness (up to $4 \AA$ RMS). The dislocations can be considered as the origin of the strain fluctuations which result in optical phonon scattering; finally, iii) tensile strain and strain fluctuations (again on the order of $10^{-2}$ ) also exist in the bulk of the underlying wafer. Every time, the strain originates from the $\mathrm{Si} / \mathrm{SiO}_{2}$ interfaces and comes from the difference in bond length and thermal expansion coefficient between the two materials. Since both the Si-overlayer and the bulk Si wafer keep the buried oxide stressed, when thinning the SOL the strain decreases in the buried oxide and increases in the handle wafer. The overall strain relaxes very slowly and extends from the BOX/wafer interface over a few micrometers range. This is in contradiction to the most common belief that the SOL/BOX/underlying handle wafers are made of very stable and strain-free materials. As a matter of fact, SOI wafers experience more equilibrium conditions which depend on every stage of the technology road.

\section{ACKNOWLEDGMENTS}

This work was supported in part by the EU commission under contract BRPR CT96 0261. We greatly thank Martin Eickoff (Daimler-Chrysler, Munich) and Y. Monteil (Université de Lyon) for sending us the different samples investigated in this work. We also thank Y. Stoemenos from Aristotle University of Thessaloniki (Greece) as well as B. Aspar and H. Moriceau from LETI-CEA (France) for expert discussions about SOI related problems. Finally, one of us (L.F.) thanks the French Ministry of Education for partial support during the course of this work.

* permanent address: Landau Institute for Theoretical Physics, Russian Academy of 
Sciences, Kosygina 2, Moscow 117 334, Russia. 


\section{REFERENCES}

[1] see, for instance, Silicon-on-insulator technology, MRS Bulletin 23, pp 13-49 (1998) and references therein.

[2] for a recent review, see: Q.-Y. Tong and U. Gosele in Semiconductor wafer bonding: Science and Technology, The Electrochemical Soc. Series (Wiley Intersciences, NY 1999).

[3] see for instance: F. Assaderaghi and G. Shahidi, Proc. "5th Int. Symp. Semicond. Wafer Bonding: Science, Technology and Applications", Honolulu, Hawaii (1999) in press.

[4] unlike SIMOX or BESOI, Unibond is not a generic name. This is a trade-mark deposited by SOITEC, Parc Technologique des Fontaines, 38190 Bernin (France); for more details see: M. Bruel, Electron. Letters 58, 1284 (1999).

[5] T.J. Letavic, H. Baumgart, P. Pinker, S. Merchant and E. Arnold, Proc. "1st Int. Symp. Semiconductor Wafer Bonding: Science, Technology and Applications", The Electrochemical Soc. Edit. p.397, Pennington, NJ (1992).

[6] K.W. Schwarz, Phys. Rev. Lett. 78, 4785 (1997).

[7] V.T. Gillard, W. D. Nix, and L. B. Freund, J. Appl. Phys. 76, 7280 (1994).

[8] A.E. Romanov, W. Pompe, S. Mathis, G.E. Beltz, and J.S. Speck, J. Appl. Phys. 85, $182(1999)$.

[9] J. Camassel, N. Planes, L. Falkovsky, H. Möller, M. Eickhoff, and G. Krötz, Electron. Letters 35, 1284 (1999).

[10] K. K. Tiong, P. M. Amirharaj, F. H. Polak, and D. E. Aspnes, Appl. Phys. Lett. 44, $122(1984)$.

[11] H. Richter, Z. P. Wang, and L. Ley, Solid State Com. 39, 625 (1981); also see: I.H. Campbell and P.M. Fauchet, Solid State Com. 58, 739 (1986). 
[12] F. Cerdeira, C. J. Buchenauer, F. H. Pollak, and M. Cardona, Phys. Rev. B 5, 580 (1972) and references therein.

[13] Z. C. Feng, W. J. Choyke, and J. A. Powell, J. Appl. Phys. 64, 6827 (1988).

[14] L. A. Falkovsky, J. M. Bluet, and J. Camassel, Phys. Rev. B 55, R14 697 (1997); also see: L.A. Falkovsky, J.M. Bluet, and J. Camassel, Phys. Rev. B57, 11283 (1998-II).

[15] M. V. Klein, in Dynamical Properties of Solids, edited by G. K. Horton and A. A. Maradudin (North - Holland, 1990), Vol. 6, pp. 65-127.

[16] L. A. Falkovsky, JETP Lett. 66, 860-868 (1997).

[17] S. Rohmfeld, M. Hundhausen, and L. Ley, Phys. Rev. B 58, 9858 (1998-I).

[18] A.A. Abrikosov, L.P. Gor'kov, I.Ye. Dzyaloshinskii, Methods of Quantum Field Theory in Statistical Physics, (Prentice-Hall, Englewood Cliffs, NJ, 1963).

[19] S. Charpenay, P. A. Rosenthal, P. R. Solomon, J. Xu, V. A. Yakovlev, L. P. Allen, M. W. Brandt, and B. Cordts, Proc. 1998 IEEE International SOI Conference, p. 43 (1998).

[20] J.F. Scott and P.S. Porto, Phys. Rev. 161, 903 (1967).

[21] W.G. Spitzer and D.A. Kleinman, Phys. Rev. 121, 1324 (1961); also see: ibid. Phys. Rev. 125, 16 (1962).

[22] A. Jayaraman, D.L. Wood and R.G. Maines, Phys. Rev. B 35, 8316 (1987).

[23] J. Camassel, A. Goullet, and J. Pascual, Phys. Rev. B 38, 8412 (1988).

[24] C.T. Kirk, Phys. Rev. B 38, 1255 (1988).

[25] E.I. Kamitsos, A.P. Patsis and G. Kordas, Phys. Rev. B 48, 12499 (1993). 


\section{Figure captions:}

Fig. 1. Schematic drawing of the Raman back-scattering geometry used in this work to investigate the strain relaxation in the silicon-on-insulator (SOI) system. The strain relaxation is probed by displacing the laser spot in the $z$-direction.

Fig. 2. Infrared reflectivity spectra collected on SOI (Unibond) material for different SOL thickness (see labels on the curves).

Figs. 3. Experimental Raman spectra collected on Si-wafers with SOL thickness $203 \mathrm{~nm}$. The SOL is separated by a $400 \mathrm{~nm}$ oxide film from the handle wafer. The distance from the interface $(5,2,1$, and $0 \mu \mathrm{m})$ of the laser spot is indicated on the curves. The solid lines are fits to Eqs. (5) - (7). The results of the fits are given in Table 1. The natural width is 3.1 $\mathrm{cm}^{-1}$ for all lines, the correlation radius $r_{0} \omega_{0} / s=16$.

Fig. 4. Same as Fig. 3 for $42 \mathrm{~nm}$ Si-overlayer thickness. In right panel, the inhomogeneous shift and linewidth are shown as a function of the frequency. The position of the line peak in fully relaxed material is shown as dash-dotted line. The inhomogeneous shift (right panel) is positive, whereas the total shift (left panel) resulting from the strain is negative.

Fig. 5. Same as Fig. 3 for $11 \mathrm{~nm}$ SOL thickness.

Fig. 6. Same as Fig. 3 for $5 \mathrm{~nm}$ SOL thickness.

Fig. 7. Same as Figs. 3-5 but now for a SIMOX wafer with $50 \mathrm{~nm}$ Si-overlayer thickness.

Results of the fits are given in Table II. The natural width is $3.4 \mathrm{~cm}^{-1}$ for all lines, the correlation radius $r_{0} \omega_{0} / s=16$.

Fig. 8. Linewidths of the two components of the optical phonon triplet in $\mathrm{Si}$ near the $\mathrm{Si} / \mathrm{SiO}_{2}$ interface as a function of the root mean square (RMS) roughness of the SOL surface. The lines are the result of the theoretical model (see Eq. (15)). The points give the width found from the fit of spectra to the theory. The corresponding RMS value is extracted from AFM measurements for the various thickness $(203,95,42,11$, and $5 \mathrm{~nm}$ ) of the Si-overlayer.

Fig. 9. Experimental Raman spectra (for Unibond wafers A) collected for different points at the BOX/wafer interface (upper line: normal situation; bottom lines: specific points dis- 
cussed in the text). The thickness of the Si-overlayer is $42 \mathrm{~nm}$.

Fig. 10. Full series of Raman spectra (for SIMOX) collected up to $5 \mu \mathrm{m}$ from a specific point at the BOX/wafers interface. The thickness of the Si-overlayer is $50 \mathrm{~nm}$.

Fig. 11. Schematic drawing of the in-plane strain in the Si-overlayer, the buried oxide and the handle silicon wafer for two different SOL thickness: nominal value $200 \mathrm{~nm}$ (solid line) and thinned SOL (dashed line). 


\section{TABLES}

TABLE I. Zone-center optical phonon frequencies and corresponding linewidths (all values are in $\mathrm{cm}^{-1}$ ) in the Unibond samples (with various top-layer thickness) in relation to the distance $z$ from the interface. The results refer to the fits with the present theory. The adjusted coupling parameters (the last column) $B_{x x}, B_{z z}$ are probabilities for the phonon intrabranch scattering by strain fluctuations. The splitting of triplet $\Gamma_{25}^{\prime}$ into the doublet $\left(\omega_{x}\right)$ and the singlet $\left(\omega_{z}\right)$ is resolved for samples with $5 \mathrm{~nm}$ and $11 \mathrm{~nm}$ top-layer thicknesses. 


\begin{tabular}{|c|c|c|c|c|}
\hline Sample & $\begin{array}{l}\text { Distance from } \\
\text { interface, } \mu \mathrm{m}\end{array}$ & $\begin{array}{l}\text { Lines } \Gamma_{25}^{\prime} \\
\omega_{z} ; \quad \omega_{x}\end{array}$ & $\begin{array}{l}\text { Linewidths } \\
\Gamma_{z} ; \quad \Gamma_{x}\end{array}$ & $\begin{array}{l}\text { Interactions } \\
B_{z z} ; \quad B_{x x}\end{array}$ \\
\hline \multirow[t]{4}{*}{$203 \mathrm{~nm}$} & 5.0 & 520.0 & 3.10 & 0. \\
\hline & 2.0 & 519.76 & 3.21 & 0.20 \\
\hline & 1.0 & 519.28 & 3.32 & 0.39 \\
\hline & 0 & 519.18 & 3.61 & 0.97 \\
\hline \multirow[t]{4}{*}{$95 \mathrm{~nm}$} & 5.0 & 520.0 & 3.10 & 0. \\
\hline & 2.0 & 520.0 & 3.10 & 0. \\
\hline & 1.0 & 520.0 & 3.32 & 0.38 \\
\hline & 0. & 519.28 & 3.69 & 1.16 \\
\hline \multirow[t]{4}{*}{$42 \mathrm{~nm}$} & 5.0 & 520.0 & 3.10 & 0. \\
\hline & 2.0 & 520.0 & 3.21 & 0.19 \\
\hline & 1.0 & $519.18 ; 516.13$ & $3.69 ; 3.26$ & $1.16 ; 0.29$ \\
\hline & 0. & $519.17 ; 519.08$ & $4.25 ; 3.42$ & $2.51 ; 0.6$ \\
\hline \multirow[t]{4}{*}{$11 \mathrm{~nm}$} & 5.0 & 520.0 & 3.10 & 0. \\
\hline & 2.0 & $520.18 ; 520.15$ & $3.32 ; 3.1$ & $0.38 ; 0$ \\
\hline & 1.0 & $520.27 ; 520.22$ & $3.86 ; 3.42$ & $1.54 ; 0.58$ \\
\hline & 0. & $519.55 ; 519.13$ & $6.23 ; 3.70$ & $9.6 ; 1.2$ \\
\hline \multirow[t]{4}{*}{$5 \mathrm{~nm}$} & 5.0 & 520.0 & 3.10 & 0. \\
\hline & 2.0 & $519.73 ; 519.70$ & $3.32 ; 3.1$ & $0.39 ; 0$. \\
\hline & 1.0 & $519.26 ; 519.27$ & $6.44 ; 3.61$ & $10.6 ; 0.96$ \\
\hline & 0. & $519.14 ; 519.00$ & $9.44 ; 4.65$ & $29.0 ; 3.67$ \\
\hline
\end{tabular}


TABLE II. Same as Table I but for a SIMOX sample with $50 \mathrm{~nm}$ top-layer thickness. The splitting of triplet was not resolved

\begin{tabular}{c|c|c|c|}
\hline \hline Distance from & Lines $\Gamma_{25}^{\prime}$ & Widths & Interactions \\
interface, $\mu \mathrm{m}$ & $\omega_{x}$ & $\Gamma_{x}$ & $B_{x x}$ \\
\hline 20.0 & 520.0 & 3.40 & 0 \\
5.0 & 520.01 & 3.50 & 0.20 \\
2.0 & 520.02 & 3.60 & 0.38 \\
1. & 519.76 & 3.70 & 0.58 \\
0. & 519.50 & 3.79 & 0.77 \\
0. & 519.30 & 3.79 & 0.77 \\
\hline \hline
\end{tabular}




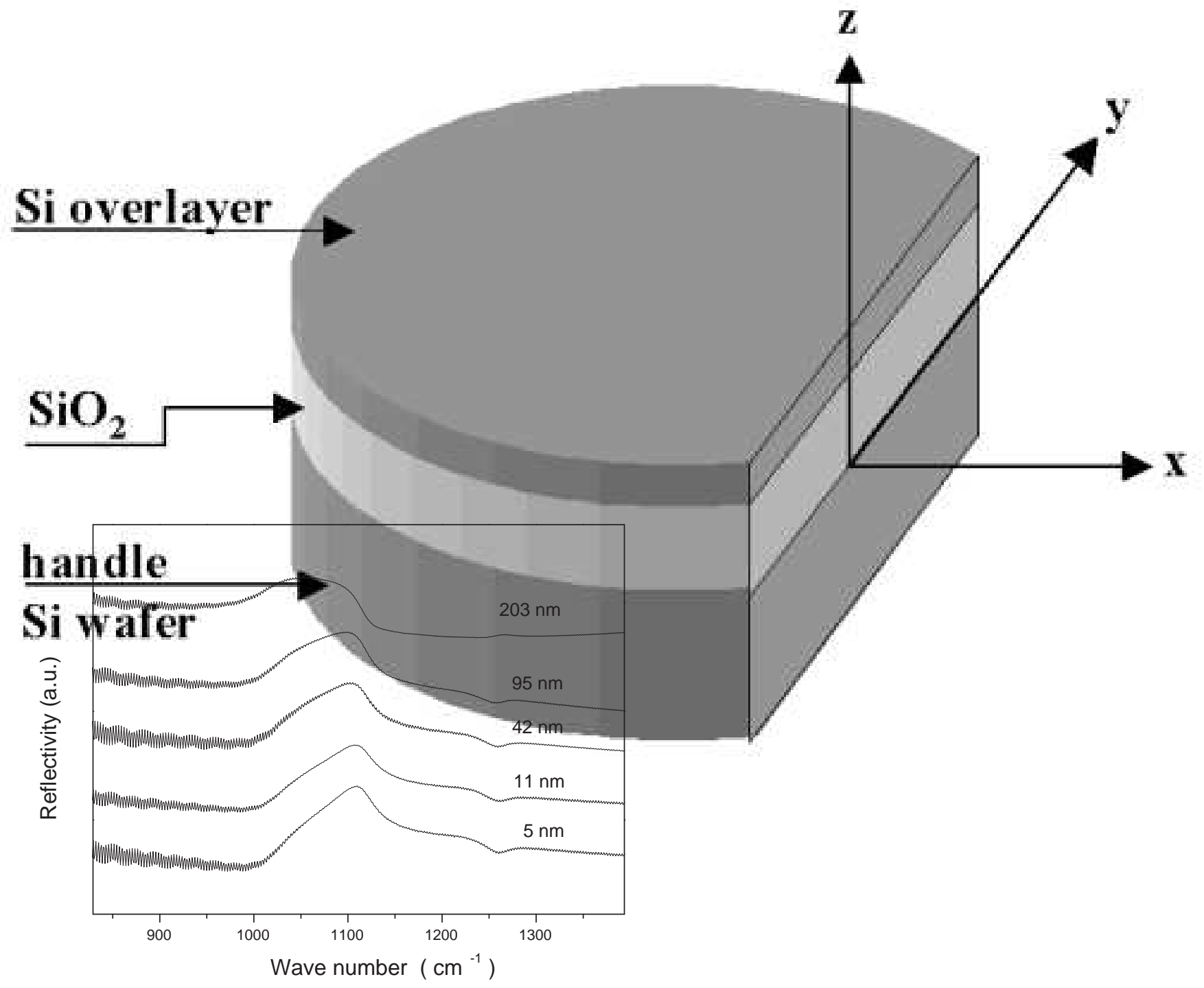




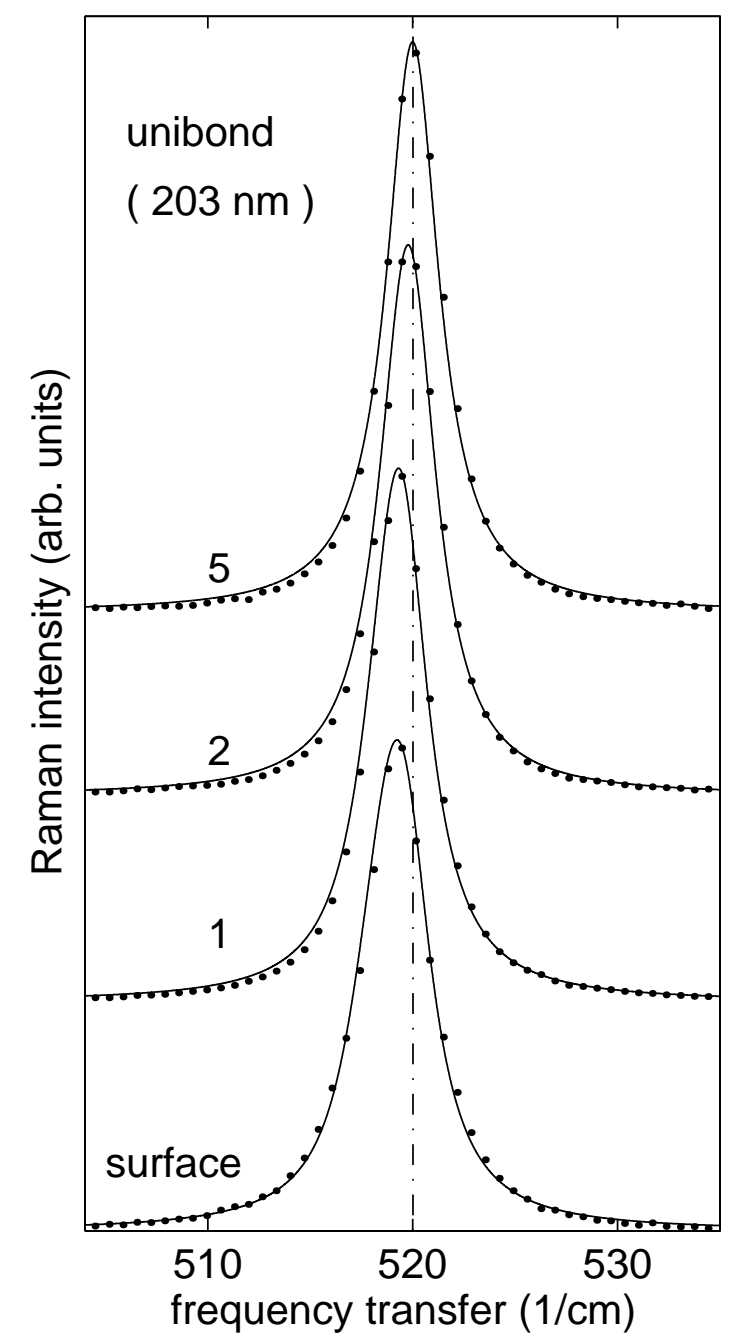



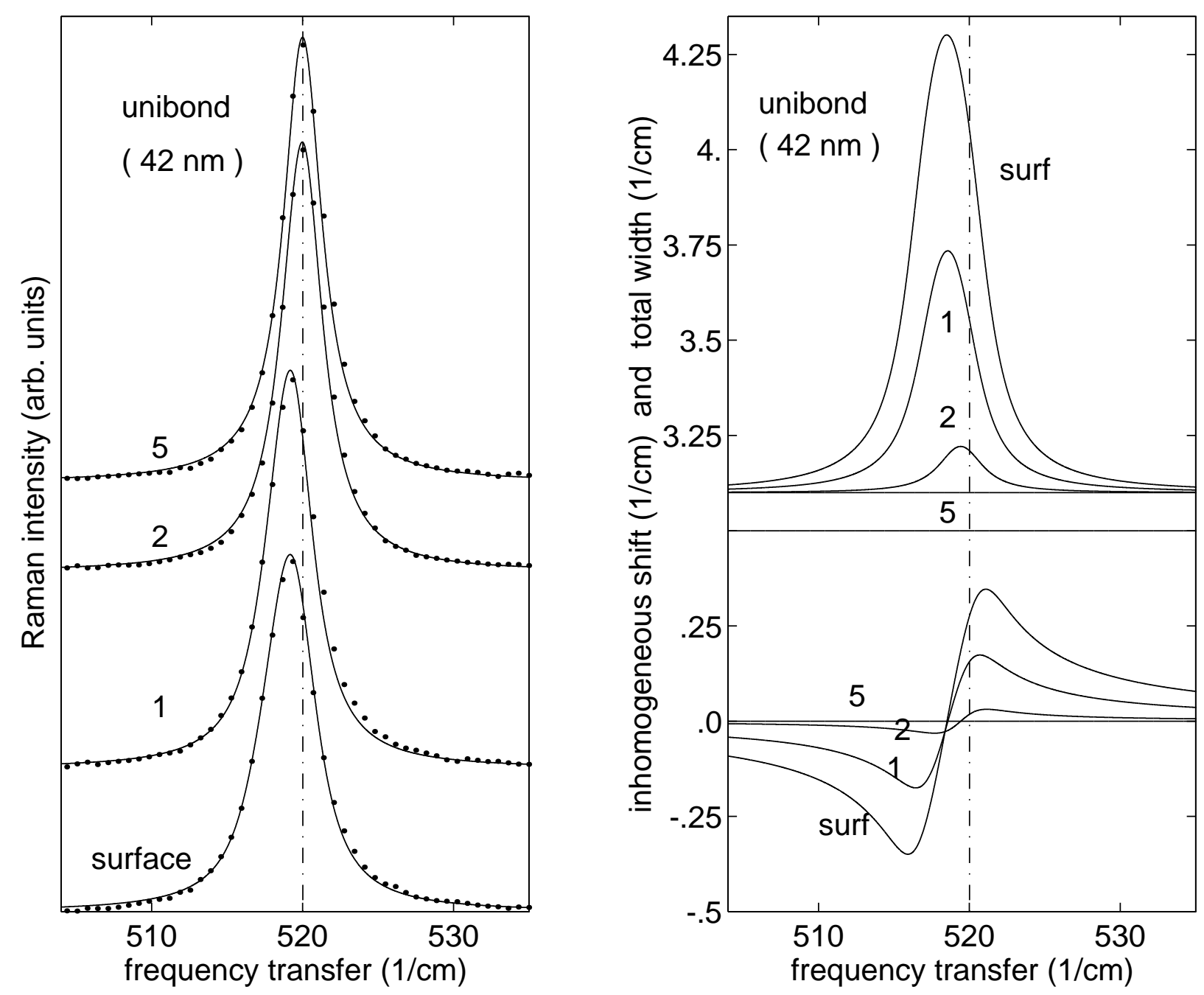


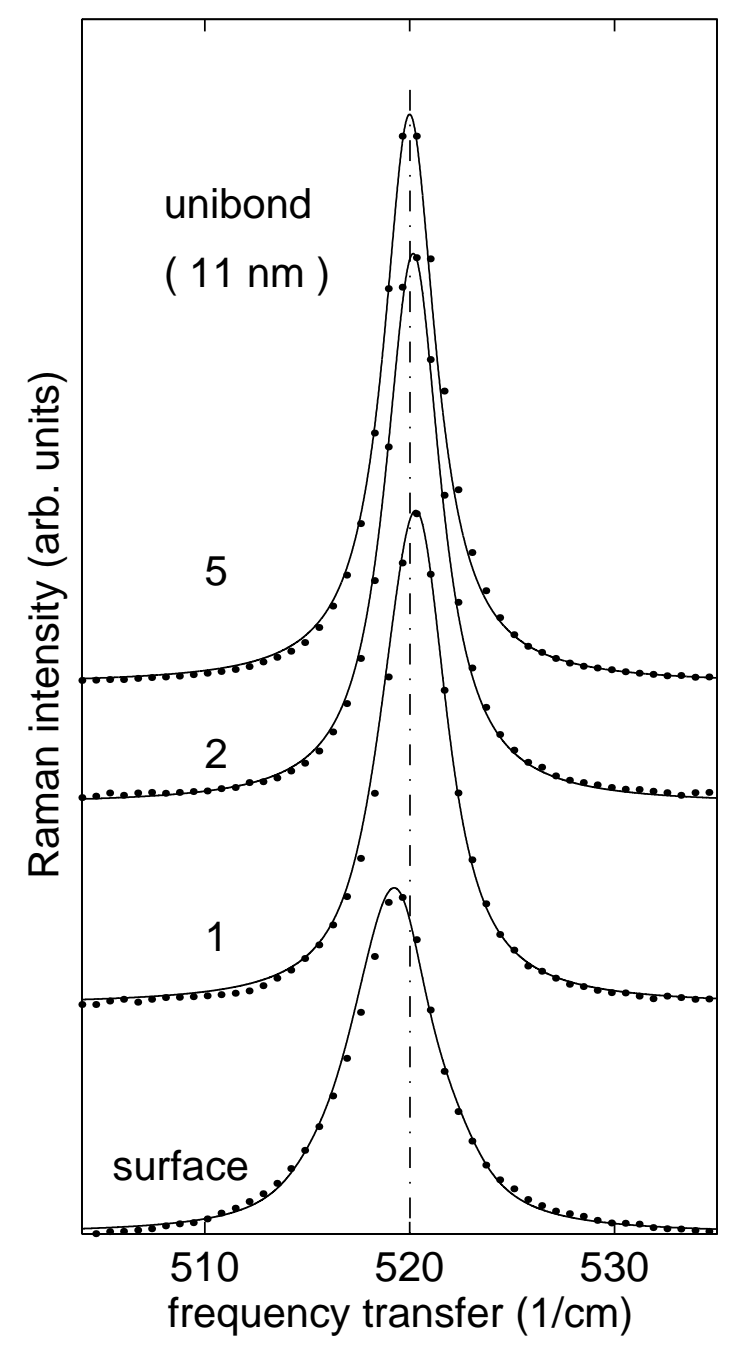




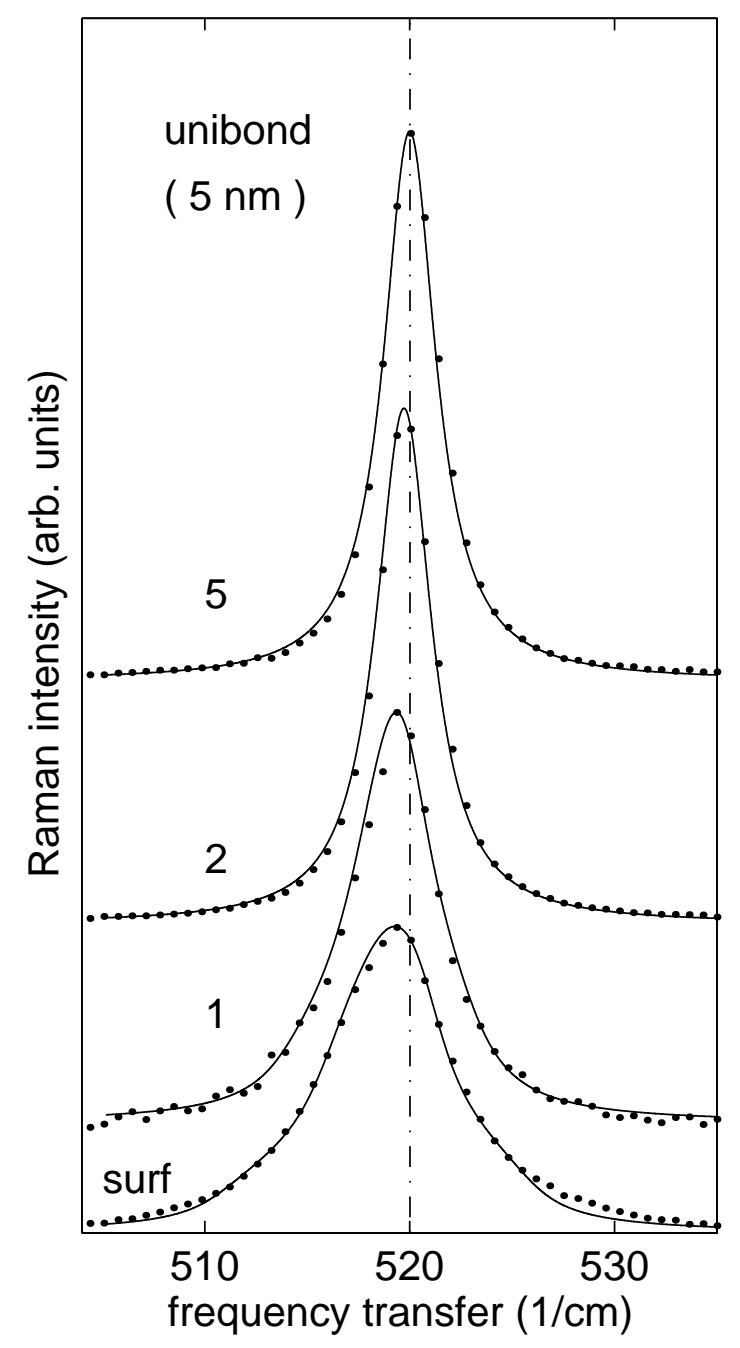




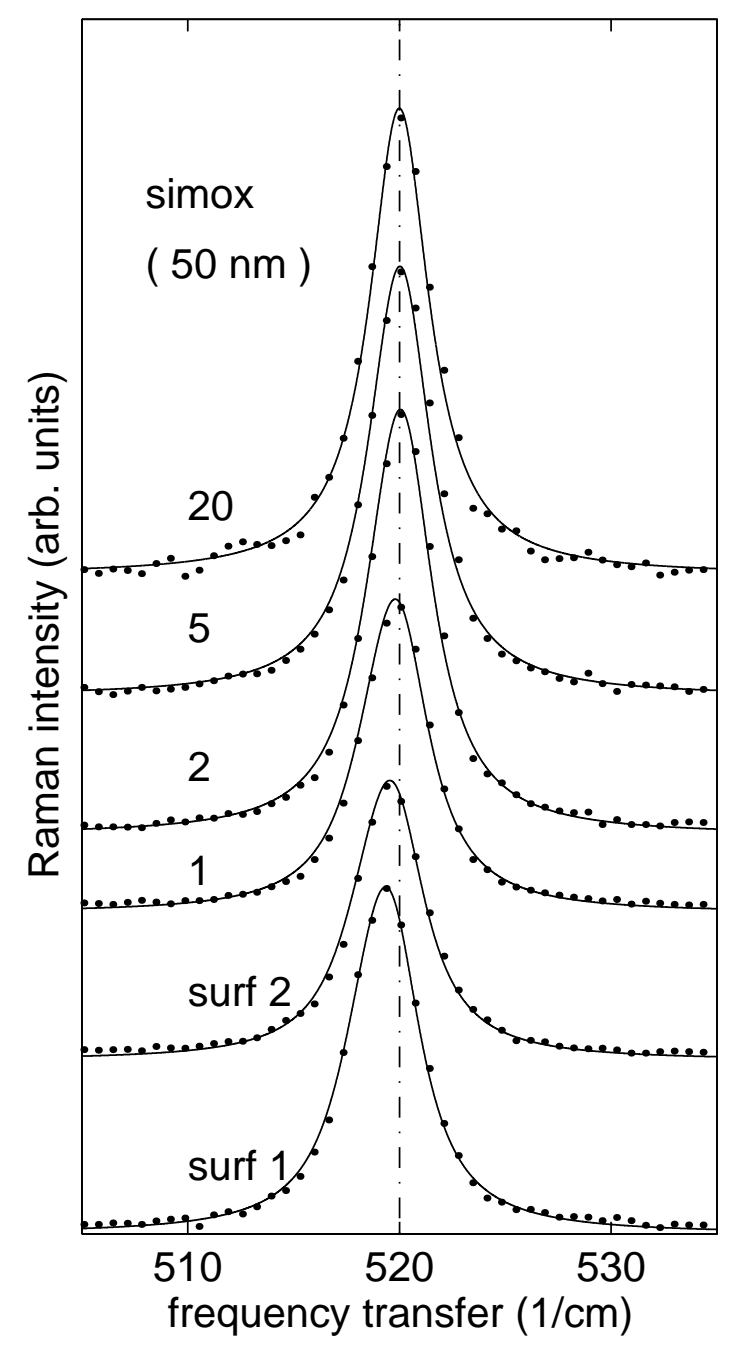




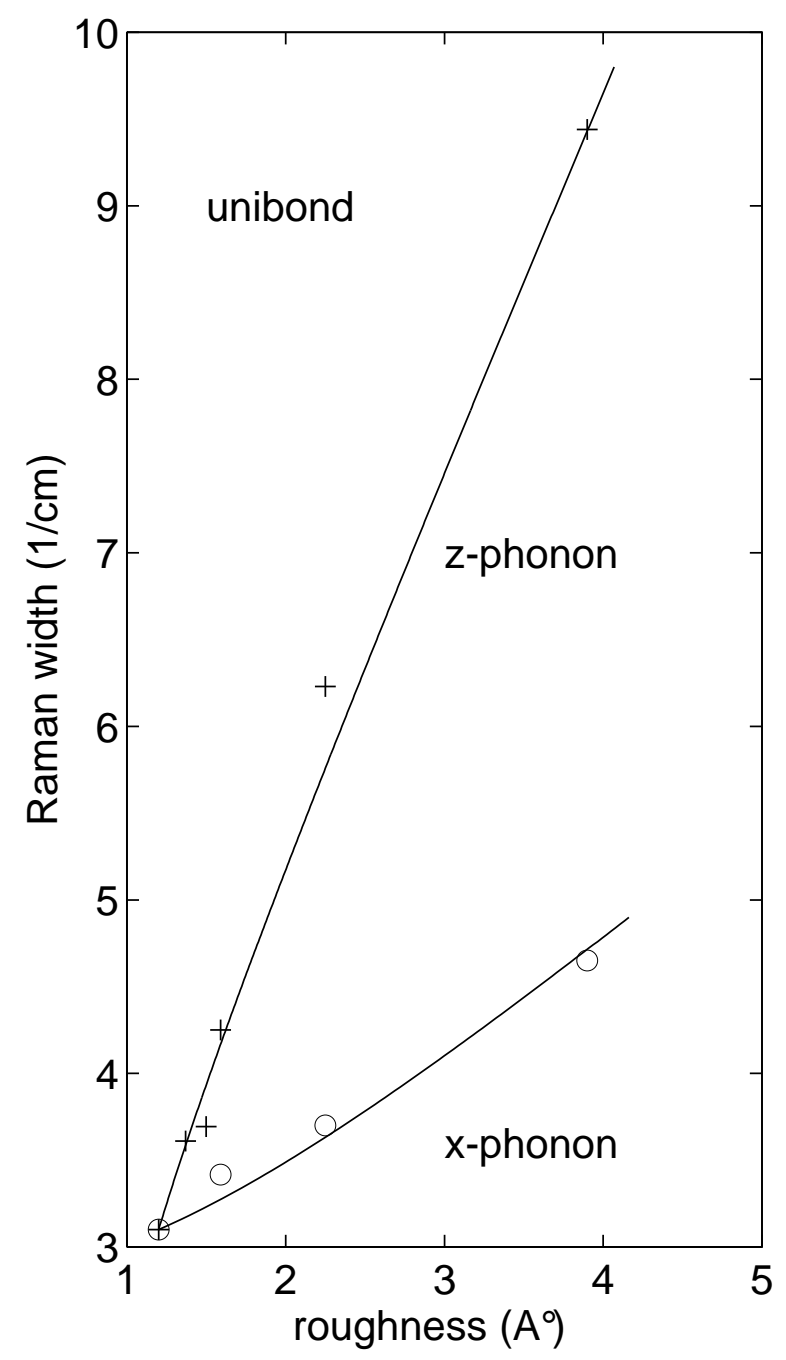




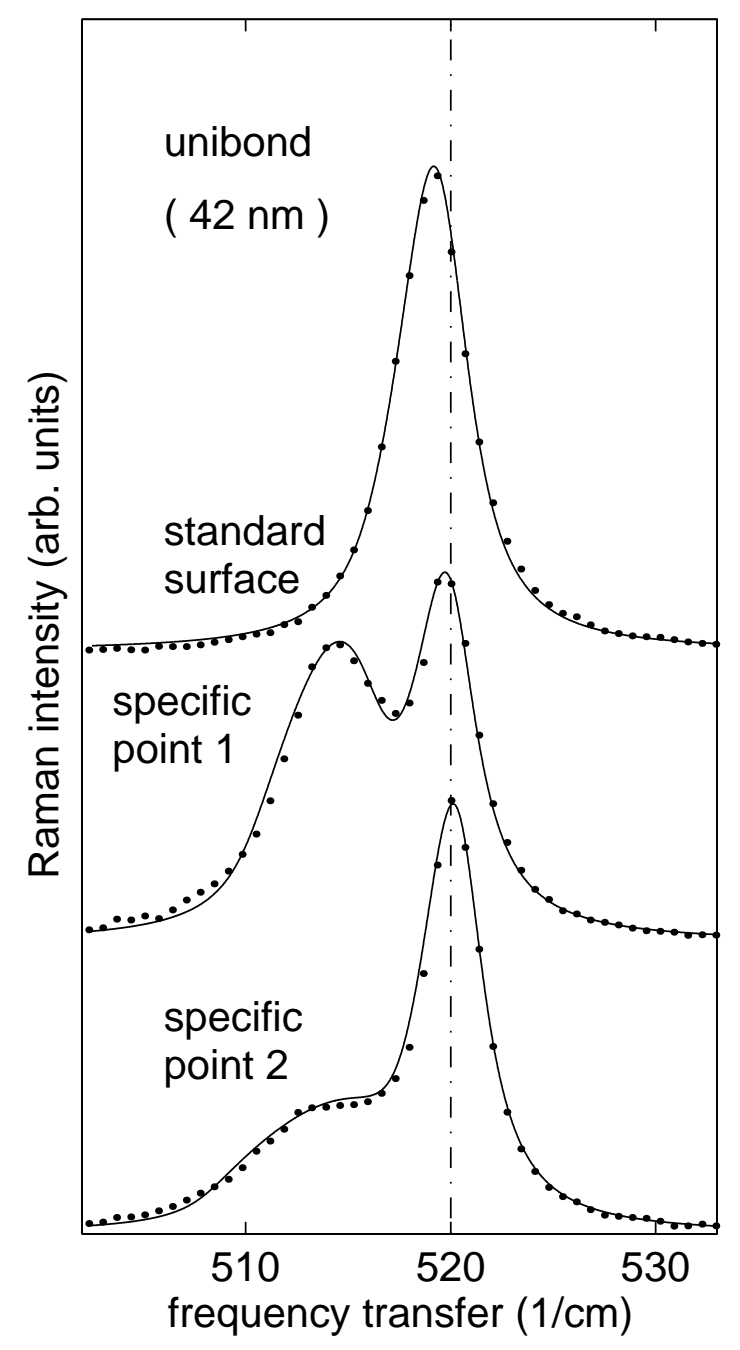




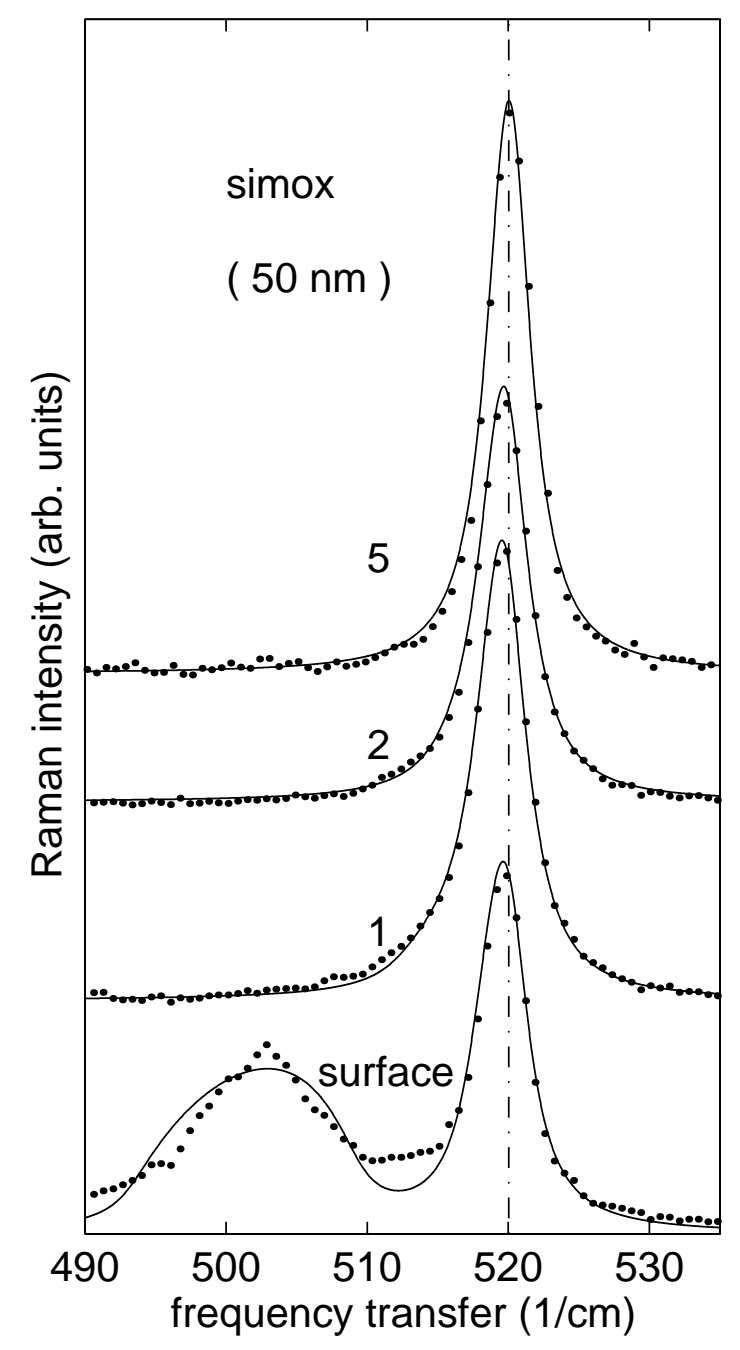

